\title{
Bayesian Analysis of the Uncertainty in Decay Lifetimes in the Case of Poor Statistics
}

\section{Application to Superheavy Nuclei}

\author{
Quentin Hourdillé $^{1,2}$, Yoann Lallouet $^{3}$, David Boilley ${ }^{\mathrm{a}, 1,2}$ \\ ${ }^{1}$ GANIL, CEA/DRF/IRFU-CNRS/IN2P3, Caen France \\ ${ }^{2}$ Normandie Univ. - Unicaen, Caen, France \\ ${ }^{3}$ Lycée Malherbe, Caen, France
}

Received: date / Accepted: date

\begin{abstract}
In case of rare decay events the lifetime or the decay width is evaluated with low statistics. The uncertainty has to be carefully evaluated. In this article, we propose a Bayesian analysis of the lifetime and decay width and derive simple formulas for their assigned values and for the limits of the credible interval. The results are applied to the decay of superheavy nuclei as an illustration.
\end{abstract}

Keywords Bayesian analysis - Decay lifetime . Superheavy Elements · Uncertainty

PACS 02.50.Cw - 06.20.Dk $\cdot 21.10 . \mathrm{Tg} \cdot 23.60 .+\mathrm{e} \cdot$ $27.90 .+\mathrm{b}$

\section{Introduction}

The heaviest elements produced so far were identified by their alpha-decay chains. Since ambiguities in assigning decay properties of nuclei often arise from too large uncertainties, these latter have to be carefully estimated. Among the experimental observables are the time lapses between the implantation of the super-heavy nucleus in the detector and the first alpha emission and then the time lapses between two successive alpha emissions. These time lapses are then used to calculate the half-lives of various isotopes of the decay chain and the dominant uncertainty in the case of few events arises from the statistical distribution of the decay process.

Being $\left\{t_{i}\right\}$, the time lapses associated to $n$ events of the decay of a given isotope, the half-life is simply $T_{1 / 2}=\ln 2 \times \tau$. The lifetime $\tau$ is generally estimated by the mean value of the observed decay times [1]

$\bar{t}=\frac{1}{n} \sum_{i=1}^{n} t_{i}$

${ }^{\mathrm{a} e-m a i l: ~ b o i l l e y @ g a n i l . f r ~}$
Given the lifetime $\tau$, the probability to observe a decay in the time interval $\left[t_{i}, t_{i}+d t_{i}\right]$ is simply

$p\left(t_{i} \mid \tau\right) d t_{i} \quad$ with $\quad p\left(t_{i} \mid \tau\right)=\frac{1}{\tau} e^{-t_{i} / \tau}$.

And the probability density function to observe the decay times $\left\{t_{i}\right\}$ associated with $n$ independent events is just the product of each individual probabilities:

$p\left(\left\{t_{i}\right\} \mid \tau\right)=\prod_{i=1}^{n} p\left(t_{i} \mid \tau\right)=\frac{1}{\tau^{n}} \prod_{i=1}^{n} e^{-t_{i} / \tau}=\frac{1}{\tau^{n}} e^{-n \bar{t} / \tau}$.

It only depends on the mean value of the observed events $\bar{t}$ and their number $n$, which means that $n \bar{t}$ is a sufficient statistic, a concept introduced by Fisher [2].

Within the so-called frequentist statistics, we generally consider that the experiment is repeated a large number of times to evaluate the uncertainty. We can easily calculate the probability density function for the mean value $\bar{t}$ which takes into account all possible $p\left(\left\{t_{i}\right\} \mid \tau\right)$ which conserve $\bar{t}$. It is given by

$$
\begin{aligned}
p(\bar{t} \mid \tau, n) & =\int_{0}^{\infty} \cdots \int_{0}^{\infty} p\left(\left\{t_{i}\right\} \mid \tau\right) \delta\left[\bar{t}-\frac{1}{n} \sum_{i=1}^{n} t_{i}\right] d t_{1} \ldots d t_{n} \\
& =\frac{n^{n+1}}{n !} \frac{\bar{t}^{n-1}}{\tau^{n}} e^{-n \bar{t} / \tau} .
\end{aligned}
$$

This equation derived in Ref. [3] assumes that the number of events $n$ is known. It gives statistical information on the mean value of the decay times, $\bar{t}$, not on the lifetime, $\tau$ which is a parameter. The latter is an estimator of the former. To determine the uncertainty in the lifetime $\tau$ we should rather determine $p(\tau \mid \bar{t}, n)$ which can be deduced from $p(\bar{t} \mid \tau, n)$ with the Bayes theorem [4].

With a large number of events, $\bar{t}$ is a good estimator of $\tau$ and the uncertainty in $\bar{t}$ can be used as the uncertainty in $\tau$. But superheavy nuclei associated to elements with an atomic number larger than 100 are 
produced in very small amounts. With low statistics, we should definitively use $p(\tau \mid \bar{t}, n)$ to estimate the lifetime $\tau$ and the uncertainty in $\tau$. This is the purpose of the present article.

\section{Frequentist confidence interval}

Characteristics of the probability density function $p(\bar{t} \mid \tau, n)$ given by Eq. (4), such as the standard deviation,

$u(\bar{t})=\frac{\tau}{\sqrt{n}}$,

depend on $\tau$, the true value of the lifetime that is unknown. However, this probability density function for $\bar{t}$ or likelihood function for $\tau$ can give constrains on the values that can be taken by $\tau$.

The maximum likelihood estimation is generally used to make inferences about the value of the parameter that is most likely to have generated the sample. Here, the maximum likelihood value of $\tau$ is $\bar{t}$.

As for the confidence interval, it can be evaluated with help of the cumulative probability function for the dimensionless variable $\theta=\bar{t} / \tau$,

$$
\begin{aligned}
P(\Theta) & =\int_{0}^{\Theta} \frac{n^{n+1}}{n !} \theta^{n-1} e^{-n \theta} d \theta, \\
& =1-e^{-n \Theta} \sum_{k=0}^{n-1} \frac{(n \Theta)^{k}}{k !},
\end{aligned}
$$

which does not explicitly depend on $\tau$. The confidence interval is not unique on a probability density function. Here we shall choose the equal-tailed interval where the probability of being below the interval is as likely as being above it. Such an interval is also called probabilistically symmetric confidence interval. The lower and upper limits of the confidence interval associated to the confidence level $\alpha, \Theta_{\alpha}^{<}$and $\Theta_{\alpha}^{>}$respectively, are determined by inverting the equations

$P\left(\Theta_{\alpha}^{<}\right)=\frac{1-\alpha}{2}$ and $P\left(\Theta_{\alpha}^{>}\right)=\frac{1+\alpha}{2}$.

The values of $\Theta_{\alpha}^{<}$and $\Theta_{\alpha}^{>}$have to be determined numerically. Eventually, the confidence interval associated to the confidence level $\alpha$ for the true value of the lifetime, $\tau$, is $\left[\bar{t} / \Theta_{\alpha}^{>}, \bar{t} / \Theta_{\alpha}^{<}\right]$.

The limits of the confidence interval in most publications reporting results on superheavy nuclei decay are determined with approximate formulas given in Ref. [3] which are reproduced from an earlier edition of the textbook [5]:

$\left[\frac{\bar{t}}{1+\frac{k_{\alpha}}{\sqrt{n}}}, \frac{\bar{t}}{1-\frac{k_{\alpha}}{\sqrt{n}}}\right]$

where $k_{\alpha}$ is the coverage factor associated to the confidence level $\alpha$ for a Gaussian density function. This approximate upper limit formula diverges for $k_{\alpha}=1$ and $n=1$. Thus, Ref. [3] recommends to only apply these approximate formulas for $n \geq 2$ when $k_{\alpha}=1$. Note that a higher number of events is necessary for larger values of the coverage factor.

\section{Bayesian evaluation of the uncertainties in the lifetime}

\subsection{Bayes theorem}

Bayes theorem [6], first applied to physics by Laplace who independently rediscovered it $[7,8]$, relates the two inverse probability density functions $p(\tau \mid \bar{t}, n)$ and $p(\bar{t} \mid \tau, n)$ :

$p(\tau \mid \bar{t}, n)=\frac{p(\tau) p(\bar{t} \mid \tau, n)}{\int_{0}^{\infty} p(\tau) p(\bar{t} \mid \tau, n) d \tau}$,

where the prior probability $p(\tau)$ includes all available information on $\tau$ before the measurement.

The main difference with the frequentist framework comes from the way the concept of probability itself is interpreted. According to the frequentist definition of probability, only repeatable random events have probabilities that are equal to the long-term frequency of occurrence of the events in question. In contrast, the Bayesian framework views probabilities as degrees of belief. The posterior probability density function $p(\tau \mid \bar{t}, n)$ obtained by applying Bayes theorem, Eq. (10), gives access to the plausibility of having a given value for the lifetime $\tau$, considering information brought by the prior probability, by the experimental results and by the model.

For a pioneering measurement, the prior should be non informative. The choice of a prior distribution in the absence of any information about the system is a delicate task and there are several possibilities [9]. We shall consider two priors that are common in a physical context. Other priors, such as conjugate priors that are an algebraic convenience, are not relevant for the present study.

\subsection{Prior}

\subsubsection{Uniform prior}

The simplest choice for the prior is to select an equiprobable probability density function for positive values of $\tau: p(\tau)=C \theta(\tau)$, where $\theta$ is the Heaviside step function. Such a prior comes from the direct application of the principle of indifference, which assigns equal probabilities to all possibilities. Then, it is easy to calculate Eq. (10) analytically. 
However, we could also have chosen the decay width $\lambda=1 / \tau$ as a parameter to be estimated. Since

$p(\tau) d \tau=-\frac{1}{\lambda^{2}} p(\lambda) d \lambda$,

a uniform probability density function for $\tau$ does not correspond to a uniform probability density function for $\lambda$. Thus, choosing $\lambda$ as a parameter to be constrained with a uniform prior leads to different results.

In Ref. [10], for example, it is suggested to retain the modes (most probable values) for both posterior probability density functions, $p(\tau \mid \bar{t}, n)$ and $p(\lambda \mid \bar{t}, n)$, because they are consistent: $\tau_{m}=1 / \lambda_{m}=\bar{t}$. However, characteristics of the two probability distributions such as the variances from the mode differ. Then, a uniform prior for $\tau$ or $\lambda$ is not suitable. We should find another prior that is invariant with the change of parameter.

\subsubsection{Jeffreys' prior}

Jeffreys' prior [11] is a uninformative prior that is invariant by the change of parameter. Considering the required invariance by changing $\tau$ into $\lambda$, we easily get that

$p(\tau) \propto \frac{\theta(\tau)}{\tau} \quad$ and $\quad p(\lambda) \propto \frac{\theta(\lambda)}{\lambda}$.

Applying Bayes theorem with such priors leads to

$p(\tau \mid \bar{t}, n)=\frac{(n \bar{t})^{n}}{(n-1) !} \frac{1}{\tau^{n+1}} e^{-n \bar{t} / \tau}$

$p(\lambda \mid \bar{t}, n)=\frac{(n \bar{t})^{n}}{(n-1) !} \lambda^{n-1} e^{-\lambda n \bar{t}}$.

These posterior density functions are also invariant by changing $\tau$ into $\lambda$. In the following we adopt Jeffreys' prior because it takes into account both our indeterminacy on the choice of the parameter and on the values it can take, thus, being the least informative prior about $\tau$.

Note that $p(\tau \mid \bar{t}, n)$ expressed just above differs from $p(\bar{t} \mid \tau, n)$ expressed by Eq. (4). Thus, the Bayesian posterior probability density function is expected to lead to results that differ from the frequentist ones.

\subsubsection{Another approach}

Experimental results give access to the complete set of decay times $\left\{t_{i}\right\}$ that carries more information than the mean value $\bar{t}$ and the number of events $n$. Instead of inverting $p(\bar{t} \mid \tau, n)$ we could have directly inverted $p\left(\left\{t_{i}\right\} \mid \tau\right)$ given by Eq. (3). Using Jeffreys' prior, Bayes theorem leads to

$p\left(\tau \mid\left\{t_{i}\right\}\right)=\frac{(n \bar{t})^{n}}{(n-1) !} \frac{1}{\tau^{n+1}} e^{-n \bar{t} / \tau}$.
Interestingly, this is exactly the same expression as for $p(\tau \mid \bar{t}, n)$. This is not a surprise: as already mentioned in the introduction, $n \bar{t}$ is a sufficient statistic, which means that this quantity carries as much information as all the results $\left\{t_{i}\right\}$ to determine the lifetime $\tau$. Actually, the exponential probability density function that characterises the decay of radioactive nuclei in Eq. (3) is a typical example of the reduction to a sufficient statistic in textbooks. See e.g. Ref. [5].

\subsection{Lifetime and uncertainty}

In Bayesian statistics, the posterior distribution contains all the available information. It is depicted in Fig. 1 for $n=3$ events.

This distribution should be used for any statistical inferences such as hypothesis testing, for example. However, a point estimate is necessary when reporting experimental results.

\subsubsection{Lifetime}

When estimating the expected value of an observable, international standards [12] usually recommend to retain the mean value because it encompasses all values. Thus, the estimated value of the lifetime could be chosen as the mean value $\bar{\tau}$ weighted by the posterior probability density function,

$\bar{\tau}=\int_{0}^{\infty} \tau p(\tau \mid \bar{t}) d \tau=\frac{n}{n-1} \bar{t}$.

As we are considering a model parameter here, we can also choose the mode or most probable value $\tau_{m}$,

$\left.\frac{d p(\tau \mid \bar{t})}{d \tau}\right|_{\tau=\tau_{m}}=0, \quad \tau_{m}=\frac{n}{n+1} \bar{t}$.

This is consistent with the choice of maximum likelihood estimate in the frequentist approach. The mode differs from the mean value.

Note that both points differ from the frequentist estimator $\bar{t}$. Of course, these differences vanish when the number of events $n$ is large. However, the ratio between these two quantities,

$$
\frac{\bar{\tau}}{\tau_{m}}=\frac{n+1}{n-1},
$$

is large when $n$ is small: it is equal to 3 when $n=2$ and to 2 when $n=3$. Even with $n=10$ the ratio is still equal to 1.22 . 

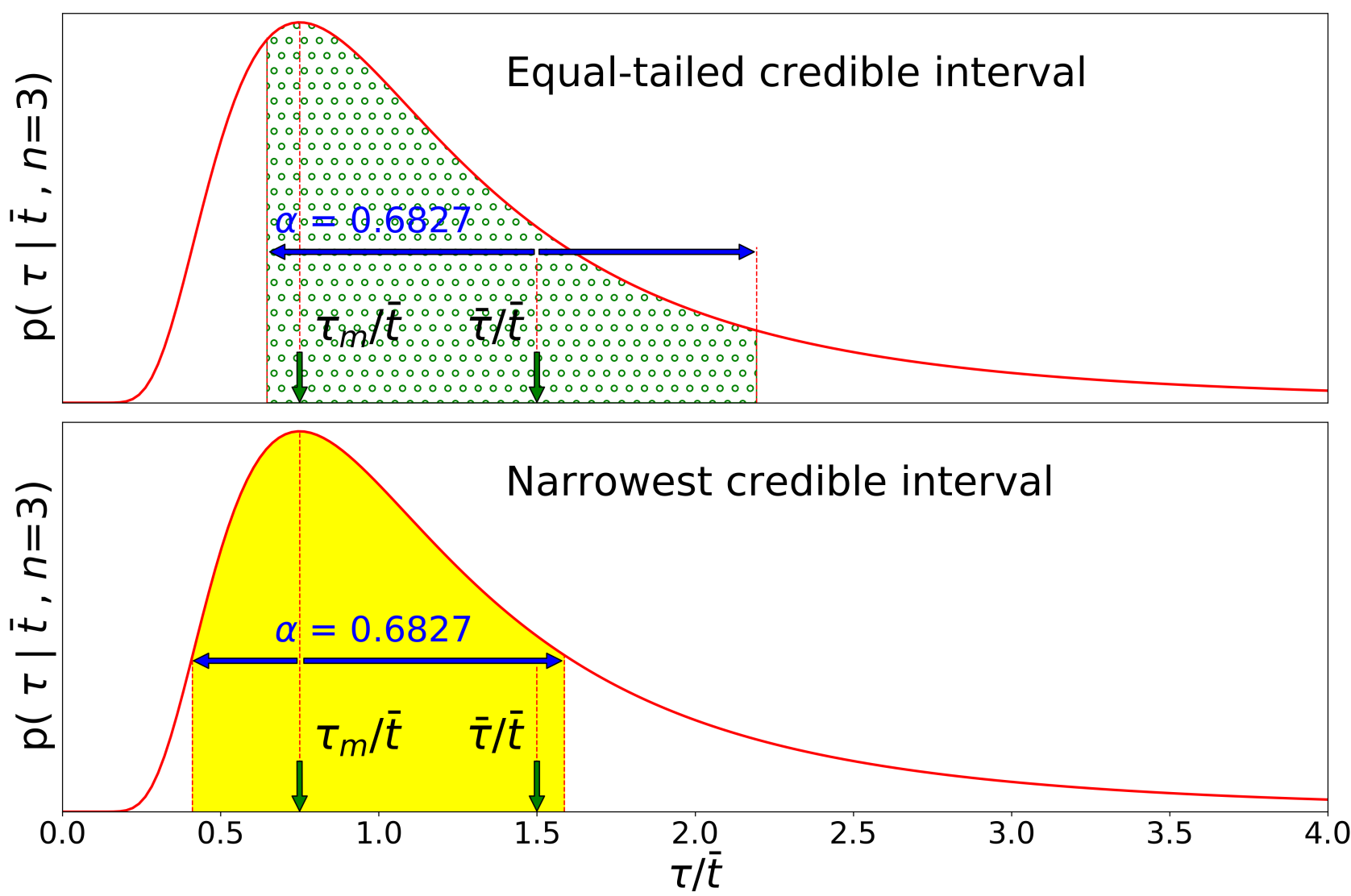

Fig. 1 Bayesian posterior probability density function $p(\tau \mid \bar{t}, n)$ for $n=3$ events in a dimensionless scale. The equal-tailed credible interval is indicated in the top panel and the narrowest credible interval is indicated in the bottom panel. Both intervals correspond to a confidence level $\alpha=0.6827$.

\subsubsection{Standard uncertainty}

The standard deviation that gives an estimate of the uncertainty can easily be calculated

$u(\tau)=\frac{\bar{\tau}}{\sqrt{n-2}}$,

which means that at least 3 events are necessary.

As the probability density function is not symmetric, the standard deviation is not sufficient to characterise the posterior probability density function and we cannot simply multiply the standard uncertainty by a coverage factor to get a coverage interval associated to a given confidence probability.

\subsubsection{Credible intervals}

Credible intervals in Bayesian statistics are analogous to confidence intervals in frequentist statistics, although they differ on a philosophical basis. In frequentist statistics $\tau$ is fixed and cannot be considered to have a distribution of possible values. A frequentist confidence interval with a confidence level $\alpha$ means that with a large number of repeated samples, $\alpha 100 \%$ of such calculated confidence intervals would include the true value of the parameter. In Bayesian statistics, $\tau$ is a random variable and the credible interval is an interval in the domain of a posterior probability distribution that includes $\alpha 100 \%$ of the values.

Credible intervals are not unique on a posterior distribution. We shall consider here two possibilities: the equal-tailed interval and the narrowest interval which is also sometimes called the highest posterior density interval. Fig. 1 shows both possibilities for $n=3$ events and a confidence level $\alpha=0.6827$. The shortest interval differs appreciably from the equal-tailed credible interval. It appears that highly probable values are excluded from the latter. Consequently, the Guide to uncertainty in measurements says that the shortest interval may be more appropriate even if its determination is not simple [12].

For both cases, we use the cumulative probability function

$P\left(\tau_{c}\right)=\int_{0}^{\tau_{c}} p(\tau \mid \bar{t}, n) d \tau=e^{-n \bar{t} / \tau_{c}} \sum_{k=0}^{n-1} \frac{1}{k !}\left(\frac{n \bar{t}}{\tau_{c}}\right)^{k}$, 
to estimate numerically the limits of the credible intervals associated to a given level of confidence.

Interestingly, we can easily show that the Bayesian and the frequentist equal-tailed intervals coincide: With a simple change of variable into $\theta=\bar{t} / \tau$ in the above equation, we can show that

$\int_{0}^{\tau_{\alpha}^{<}} p(\tau \mid \bar{t}, n) d \tau=\frac{1-\alpha}{2}$

is equivalent to

$\int_{0}^{\bar{t} / \tau_{\alpha}^{<}} \frac{n^{n+1}}{n !} \theta^{n-1} e^{-n \theta} d \theta=\frac{1+\alpha}{2}$,

which gives access to frequentist limit. Similarly for the other limit.

\subsection{Application to the alpha decay of ${ }^{278} \mathrm{Nh}$}

As an example, we consider the three events of alphadecay of the ${ }^{278} \mathrm{Nh}$ for which three times were measured: $t_{1}=0.344 \mathrm{~ms}, t_{2}=4.93 \mathrm{~ms}$ and $t_{3}=0.667 \mathrm{~ms}$ [13]. The mean value is $\bar{t}=1.98 \mathrm{~ms}$ and corresponds to the frequentist estimate of the lifetime. The Bayesian posterior probability density function $p(\tau \mid \bar{t}, n)$ for $n=3$ events is depicted in Fig. 1 in a dimensionless scale. The most probable value for the lifetime of ${ }^{278} \mathrm{Nh}$ is $\tau_{m}=1.49 \mathrm{~ms}$ and the mean value of posterior probability density function is $\bar{\tau}=2.97 \mathrm{~ms}$.

The approximate frequentist confidence interval given by Eq. (9), with a coverage factor $k_{\alpha}=1$, is $[1.3 ; 4.7]$ ms. However, with $n=3$, these approximate formulas are not accurate. The exact equal-tailed frequentist interval deduced from Eq. (8) for a confidence level $\alpha=0.6827$ is $[1.28 ; 4.34] \mathrm{ms}$. As a comparison, we shall consider the Bayesian credible intervals with the same confidence level $\alpha=0.6827$ depicted in Fig. 1. Applied to the three decay events of ${ }^{278} \mathrm{Nh}$, the equal-tailed interval $[1.28 ; 4.34] \mathrm{ms}$ and coincides with the frequentist one. The narrowest credible interval is $[0.81 ; 3.14] \mathrm{ms}$.

We shall first join the mean value and the equaltailed interval to compare the results to the frequentist approach: with such a choice, the alpha decay of ${ }^{278} \mathrm{Nh}$ has a lifetime equal to

$\bar{\tau}=3.0_{-1.7}^{+1.4} \mathrm{~ms}, \quad$ with $\quad \alpha=0.6827$,

which differs with the frequentist result published in Ref. [13], 2.0 $0_{-0.7}^{+2.7}$, with approximate values for the limit of the confidence interval. We rather recommend the choice of the mode with the shortest credible interval. A Bayesian analysis with Jeffreys' prior would finally give for the alpha decay of ${ }^{278} \mathrm{Nh}$, a lifetime equal to

$\tau_{m}=1.5_{-0.7}^{+1.7} \mathrm{~ms}, \quad$ with $\quad \alpha=0.6827$.
Note that we could have also combined the mean value with the shortest interval as it is done in the examples of the Guide to uncertainty in measurement [12],

We can also consider the case of ${ }^{262} \mathrm{Db}$, one of the decay products of the ${ }^{278} \mathrm{Nh}$ that was used for identification in Ref. [13]. Two spontaneous fission events where observed with decay times $t_{1}=40.9 \mathrm{~s}$ and $t_{2}=0.787$ $\mathrm{s}$ and an alpha emission with a decay time $t_{3}=126 \mathrm{~s}$. Selecting the mode and the shortest credible interval, these three events lead to a Bayesian lifetime equal to $\tau_{m}=42_{-19}^{+47} \mathrm{~s}$ with a confidence level $\alpha=0.6827$. This also differs from the frequentist result published in Ref. [13]. But, this representation of the results also leads to a compatibility with the reference value given in Ref. [14]: $\tau_{r}=(49 \pm 6)$ s. The conclusion would have been the same with the equal-tailed credible interval.

\section{Adding new data}

If new data become available, it is easy to include them by applying once again Bayes theorem. The posterior obtained so far becomes the new prior. Denoting $\left\{t_{j}^{\prime}\right\}$ the set of $n^{\prime}$ new results with a mean value $\bar{t}^{\prime}$, the updated posterior,

$p\left(\tau \mid\left\{t_{i}, t_{j}^{\prime}\right\}\right)=\frac{\left(n \bar{t}+n^{\prime} \bar{t}^{\prime}\right)^{n+n^{\prime}}}{\left(n+n^{\prime}-1\right) !} \frac{1}{\tau^{n+n^{\prime}+1}} e^{-\left(n \bar{t}+n^{\prime} \bar{t}^{\prime}\right) / \tau}$,

is identical to the one obtained with a single set of $n+n^{\prime}$ decay times.

\section{Summary}

Bayesian analysis is preferred when we have to rely on a very small number of events to estimate the values that can be taken by an observable. The posterior probability density function, which combines the prior information with the information provided by the experiment, contains all available information on these values. In the case of the lifetime of a radioactive nucleus, the posterior probability density function can be calculated analytically and it is given by Eq. (15). It provides the plausibility of having a given value for the lifetime. As $n \bar{t}$ is a sufficient statistic, $n$ and $\bar{t}$ are the only quantities that need to be reported to determine the posterior probability density function that should be used for any evaluation.

Traditionally, experimental reports are reduced to an expected value and a coverage interval or an uncertainty. A simple formula is available for the mean posterior value, Eq. (16), and the mode, Eq. (17). The limits of the credible intervals have to be determined numerically. Fig 2 shows the evolution of the shortest and 

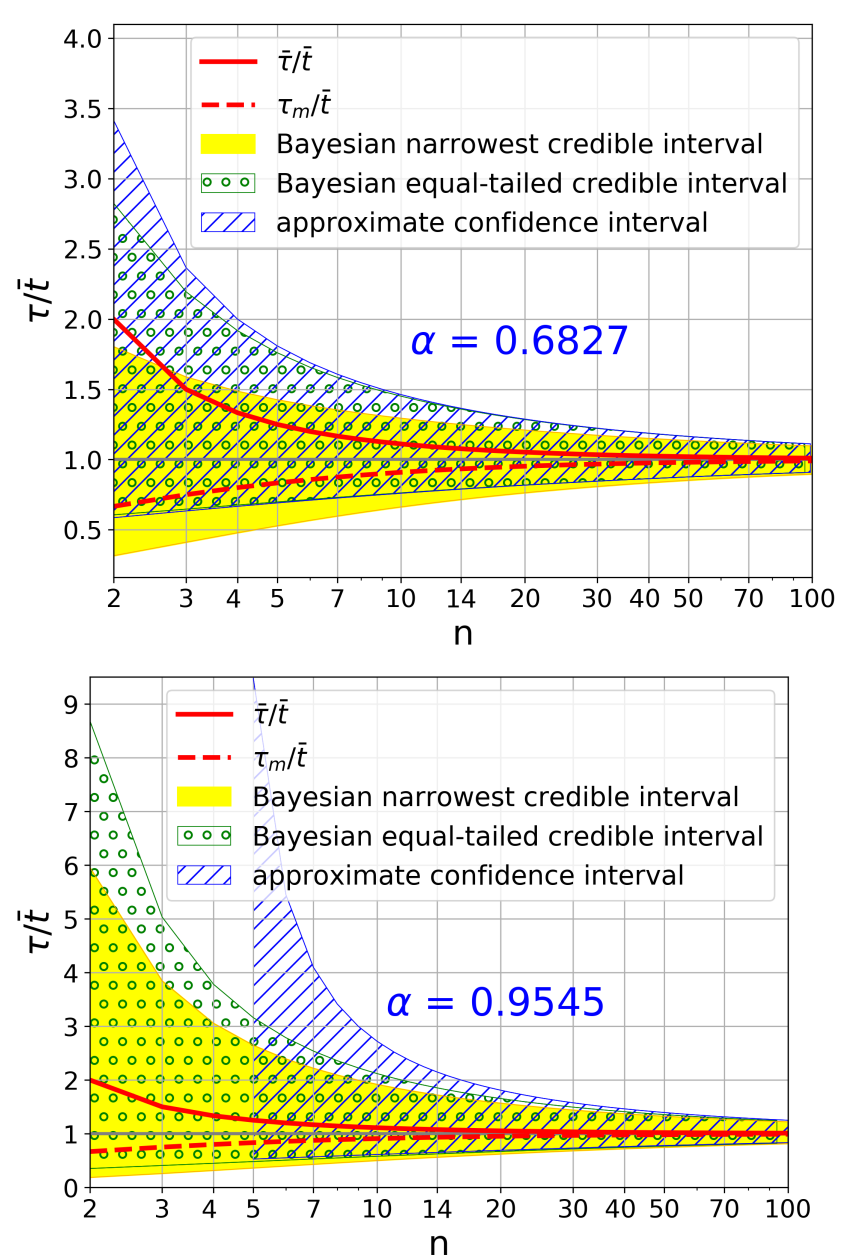

Fig. 2 Narrowest and equal tailed Bayesian credible intervals as a function of the number of events $n$ for the dimensionless variable $\tau / \bar{t}$. the approximate frequentist interval from Eq. (9) is also represented, as well as the mean value and the mode. The top panel corresponds to the level of confidence $\alpha=0.6827$ and the coverage factor $k_{\alpha}=1$. The bottom panel corresponds to the level of confidence $\alpha=0.9545$ and the coverage factor $k_{\alpha}=2$.

equal-tailed credible intervals as a function of the number of events $n$ for two confidence levels, $\alpha=0.6827$ and $\alpha=0.9545$. Mean posterior values and most probable posterior values are also plotted.

When the number of events is large enough both credible intervals coincide. However, they differ in an appreciate manner for low statistics. Note that for $n=$ 2 , the 0.6827 shortest interval does not include the mean posterior value! And the mode is very close to the lower limit of the equal-tailed credible interval, which means that the latter does not include highly probable values. Thus, the mode associated to the shortest credible interval should be selected. Whatever the choice, the report should clearly indicate which value and which interval have been selected because a reader interested in
Table 1 Limits of equal tailed credible interval as a function of the number of events $n$ for two confidence levels $\alpha=0.6827$ and $\alpha=0.9545$.

\begin{tabular}{|c|c|c|c|c|}
\hline$n$ & $\tau_{0.6827}^{<} / \bar{t}$ & $\tau_{0.6827}^{>} / \bar{t}$ & $\tau_{0.9545}^{<} / \bar{t}$ & $\tau_{0.9545}^{>} / \bar{t}$ \\
\hline 2 & 0.6061 & 2.824 & 0.3519 & 8.690 \\
\hline 3 & 0.6468 & 2.194 & 0.4082 & 5.031 \\
\hline 4 & 0.6759 & 1.918 & 0.4494 & 3.781 \\
\hline 5 & 0.6980 & 1.760 & 0.4814 & 3.159 \\
\hline 6 & 0.7158 & 1.657 & 0.5076 & 2.787 \\
\hline 7 & 0.7304 & 1.584 & 0.5295 & 2.538 \\
\hline 8 & 0.7428 & 1.529 & 0.5483 & 2.359 \\
\hline 9 & 0.7534 & 1.486 & 0.5647 & 2.225 \\
\hline 10 & 0.7628 & 1.451 & 0.5792 & 2.119 \\
\hline 10 & 0.7628 & 1.451 & 0.5792 & 2.119 \\
\hline 11 & 0.7710 & 1.422 & 0.5921 & 2.034 \\
\hline 12 & 0.7784 & 1.398 & 0.6038 & 1.963 \\
\hline 13 & 0.7850 & 1.377 & 0.6144 & 1.903 \\
\hline 14 & 0.7911 & 1.359 & 0.6329 & 1.853 \\
\hline 15 & 0.7966 & 1.343 & 0.6412 & 1.809 \\
\hline 16 & 0.8016 & 1.329 & 0.6412 & 1.771 \\
\hline 17 & 0.8063 & 1.316 & 0.6488 & 1.737 \\
\hline 18 & 0.8107 & 1.305 & 0.6559 & 1.706 \\
\hline 19 & 0.8147 & 1.294 & 0.6626 & 1.679 \\
\hline 20 & 0.8185 & 1.285 & 0.6688 & 1.654 \\
\hline
\end{tabular}

the results for statistical inferences would need $n$ and $\bar{t}$ to characterise the posterior probability density function.

The equal-tailed frequentist confidence interval coincides with the Bayesian equal-tailed credible interval. The approximate frequentist interval limits given by Eq. (9) are also represented in Fig. 2. It appears that the simple approximate formulas can be used to estimate the limits of the Bayesian equal-tailed credible interval when the number of events $n \geq 10$ and $\alpha=0.6827$. However, these approximate limits only join the limits of the shortest interval when $n \geq 40$. When the approximate formulas cannot be used, the lower and upper limits of the credible interval for the first values of the number of events $n$ have to be tabulated. Table 1 provides the limits of the equal tailed credible interval for two confidence levels, $\alpha=0.6827$ and $\alpha=0.9545$ up to $n=20$. And Table 2 provides the limits of the narrowest credible interval for the same confidence levels up to $n=50$.

Since the change of variables from the tabulated values to real values of the limits of the credible interval is linear, the interval remains the narrowest. 
Table 2 Limits of narrowest credible interval as a function of the number of events $n$ for two confidence levels $\alpha=0.6827$ and $\alpha=0.9545$.

\begin{tabular}{|c|c|c|c|c|}
\hline$n$ & $\tau_{0.6827}^{<} / \bar{t}$ & $\tau_{0.6827}^{>} / \bar{t}$ & $\tau_{0.9545}^{<} / \bar{t}$ & $\tau_{0.9545}^{>} / \bar{t}$ \\
\hline 2 & 0.3156 & 1.803 & 0.1864 & 5.954 \\
\hline 3 & 0.4116 & 1.588 & 0.2595 & 3.841 \\
\hline 4 & 0.4787 & 1.486 & 0.3163 & 3.053 \\
\hline 5 & 0.5285 & 1.425 & 0.3617 & 2.643 \\
\hline 6 & 0.5670 & 1.384 & 0.3989 & 2.389 \\
\hline 7 & 0.5979 & 1.353 & 0.4301 & 2.216 \\
\hline 8 & 0.6233 & 1.329 & 0.4568 & 2.090 \\
\hline 9 & 0.6446 & 1.310 & 0.4799 & 1.993 \\
\hline 10 & 0.6628 & 1.294 & 0.5002 & 1.916 \\
\hline 11 & 0.6786 & 1.280 & 0.5182 & 1.853 \\
\hline 12 & 0.6924 & 1.269 & 0.5343 & 1.801 \\
\hline 13 & 0.7047 & 1.258 & 0.5488 & 1.757 \\
\hline 14 & 0.7156 & 1.249 & 0.5620 & 1.718 \\
\hline 15 & 0.7255 & 1.241 & 0.5740 & 1.685 \\
\hline 16 & 0.7344 & 1.233 & 0.5850 & 1.656 \\
\hline 17 & 0.7426 & 1.227 & 0.5952 & 1.630 \\
\hline 18 & 0.7500 & 1.220 & 0.6047 & 1.606 \\
\hline 19 & 0.7569 & 1.215 & 0.6135 & 1.585 \\
\hline 20 & 0.7632 & 1.210 & 0.6217 & 1.566 \\
\hline 21 & 0.7691 & 1.205 & 0.6294 & 1.548 \\
\hline 22 & 0.7746 & 1.200 & 0.6366 & 1.532 \\
\hline 23 & 0.7797 & 1.196 & 0.6434 & 1.517 \\
\hline 24 & 0.7845 & 1.192 & 0.6498 & 1.504 \\
\hline 25 & 0.7890 & 1.188 & 0.6558 & 1.491 \\
\hline 26 & 0.7932 & 1.185 & 0.6616 & 1.479 \\
\hline 27 & 0.7972 & 1.182 & 0.6670 & 1.468 \\
\hline 28 & 0.8010 & 1.178 & 0.6722 & 1.457 \\
\hline 29 & 0.8046 & 1.175 & 0.6771 & 1.448 \\
\hline 30 & 0.8080 & 1.173 & 0.6819 & 1.438 \\
\hline 31 & 0.8112 & 1.170 & 0.6864 & 1.430 \\
\hline 32 & 0.8143 & 1.167 & 0.6907 & 1.422 \\
\hline 33 & 0.8172 & 1.165 & 0.6948 & 1.414 \\
\hline 34 & 0.8200 & 1.163 & 0.6988 & 1.406 \\
\hline 35 & 0.8227 & 1.160 & 0.7026 & 1.399 \\
\hline 36 & 0.8252 & 1.158 & 0.7062 & 1.393 \\
\hline 37 & 0.8277 & 1.156 & 0.7098 & 1.386 \\
\hline 38 & 0.8301 & 1.154 & 0.7132 & 1.380 \\
\hline 39 & 0.8323 & 1.152 & 0.7164 & 1.375 \\
\hline 40 & 0.8345 & 1.150 & 0.7196 & 1.369 \\
\hline 41 & 0.8366 & 1.149 & 0.7226 & 1.364 \\
\hline 42 & 0.8386 & 1.147 & 0.7256 & 1.358 \\
\hline 43 & 0.8406 & 1.145 & 0.7285 & 1.354 \\
\hline 44 & 0.8425 & 1.144 & 0.7312 & 1.349 \\
\hline 45 & 0.8443 & 1.142 & 0.7339 & 1.344 \\
\hline 46 & 0.8461 & 1.141 & 0.7365 & 1.340 \\
\hline 47 & 0.8478 & 1.139 & 0.7390 & 1.336 \\
\hline 48 & 0.8494 & 1.138 & 0.7415 & 1.332 \\
\hline 49 & 0.8510 & 1.136 & 0.7438 & 1.328 \\
\hline 50 & 0.8525 & 1.135 & 0.7462 & 1.324 \\
\hline
\end{tabular}

Acknowledgements The authors thank Dieter Ackermann and Christelle Stodel for their interest in this work and comments.

\section{References}

1. K.H. Schmidt, The European Physical Journal A 8(1), 141 (2000). DOI 10.1007/s100500070129. URL https: //doi.org/10.1007/s100500070129

2. R.A. Fisher, Philosophical Transactions of the Royal Society of London A 222, 309 (1922). URL https: //doi.org/10.1098/rsta.1922.0009

3. K.H. Schmidt, C.C. Sahm, K. Pielenz, H.G. Clerc, Zeitschrift für Physik A Atoms and Nuclei 316(1), 19 (1984). DOI 10.1007/BF01415656. URL https://doi. org/10.1007/BF01415656

4. D. Sivia, J. Skilling, Data Analysis: A Bayesian Tutorial (Oxford University Press, 2006)

5. W. Eadie, D. Drijard, F.E. James, M. Roos, B. Sadoulet, Statistical methods in experimental physics (NorthHolland, 1988)

6. M. Bayes, M. Price, Philosophical Transactions (16831775) 53, 370 (1763). URL http://www.jstor.org/ stable/105741

7. P.S. Laplace, Euvres complètes 8, 27 (1774). URL https://gallica.bnf.fr/ark:/12148/bpt6k77596b. image.f32. langFR

8. P.S. Laplace, Statist. Sci. 1(3), 364 (1986). DOI 10. 1214/ss/1177013621. URL https://doi.org/10.1214/ ss/1177013621

9. J.P. Laedermann, J.F. Valley, F.O. Bochud, Metrologia 42(5), 442 (2005). DOI 10.1088/0026-1394/42/5/015. URL https://doi.org/10.1088/0026-1394/42/5/015

10. M. Annis, W. Cheston, H. Primakoff, Rev. Mod. Phys. 25, 818 (1953). DOI 10.1103/RevModPhys.25.818. URL https://link.aps.org/doi/10.1103/RevModPhys. 25.818

11. H. Jeffreys, Proc. R. Soc. Lond. A 186, 453 (1946). URL https://doi.org/10.1098/rspa.1946.0056

12. W.G. 1, Joint Committee for Guides in Metrology 101 (2008). URL https://www.bipm.org/en/publications/ guides/gum.html

13. K. Morita, K. Morimoto, D. Kaji, H. Haba, K. Ozeki, Y. Kudou, T. Sumita, Y. Wakabayashi, A. Yoneda, K. Tanaka, S. Yamaki, R. Sakai, T. Akiyama, S.i. Goto, H. Hasebe, M. Huang, T. Huang, E. Ideguchi, Y. Kasamatsu, K. Katori, Y. Kariya, H. Kikunaga, H. Koura, H. Kudo, A. Mashiko, K. Mayama, S.i. Mitsuoka, T. Moriya, M. Murakami, H. Murayama, S. Namai, A. Ozawa, N. Sato, K. Sueki, M. Takeyama, F. Tokanai, T. Yamaguchi, A. Yoshida, Journal of the Physical Society of Japan 81(10), 103201 (2012). DOI 10. 1143/JPSJ.81.103201. URL https://doi.org/10.1143/ JPSJ.81.103201

14. M. Schmorak, Nuclear Data Sheets 57(4), 515 (1989). URL https://doi.org/10.1016/S0090-3752(89) 80092-4 